\title{
Antioxidant and Antimicrobial Activity of Ophiurasaponin Extracted from Ophiopholis mirabilis
}

Rongzhen Wang, Xiaoyu Xue, Jingrong Zhen, and Chenghua Guo

School of Life Sciences, Yantai University, Yantai 264005, China

Correspondence should be addressed to Chenghua Guo; gch@ytu.edu.cn

Received 1 April 2014; Revised 15 May 2014; Accepted 19 May 2014; Published 4 June 2014

Academic Editor: Wang Chunming

Copyright (c) 2014 Rongzhen Wang et al. This is an open access article distributed under the Creative Commons Attribution License, which permits unrestricted use, distribution, and reproduction in any medium, provided the original work is properly cited.

The aim of this study was to analyze antioxidant and antimicrobial activity of ophiurasaponin extracted from Ophiopholis mirabilis (overall). Ophiurasaponin was extracted with solvent extraction and purified through AB-8 macroporous resin, silica gel column chromatography, Sephadex LH-20 gel column chromatography, and C18 ODS column chromatography. The antioxidant activity of ophiurasaponin was detected by the chemiluminescence assay. The paper filtering method and the modified agar dilution method were used to determine antimicrobial activity. The results showed that the content of crude ophiurasaponin and the refined ophiurasaponin was $46.75 \%$ and $96.72 \%$, respectively. The values of the $\mathrm{IC}_{50}$ of hydroxyl-radicals, superoxide anions, and peroxide were $25.54 \mathrm{mg} / \mathrm{mL}, 9.98 \mathrm{mg} / \mathrm{mL}$, and $1.37 \mathrm{mg} / \mathrm{mL}$, respectively. The refined ophiurasaponin had a good inhibitory effect on Escherichia coli, Bacillus subtilis, Staphylococcus aureus, Aerobacter aerogenes, and Proteusbacillus vulgaris, and the minimum inhibitory concentration (MIC) was $0.0443 \mathrm{mg} / \mathrm{mL}$. In conclusion, ophiurasaponin from Ophiopholis mirabilis had obvious antioxidant activities and antimicrobial activities which could provide the theoretical basis for further research and development of antioxidant and antimicrobial marine drugs.

\section{Introduction}

Ophiopholis mirabilis is normally restricted to the shallow water of the northern and central Yellow Sea of China, such as along the coast of Dalian, Qingdao, and Yantai. Zoologically it belongs to Echinodermata, Ophiuroidea, Gnathophiurina, Ophiactidae, Ophiopholis Muller, and Troschel [1]. In this experiment, Ophiopholis mirabilis was harvested from Changdao archipelago (longitude $120^{\circ} 35^{\prime} 28^{\prime \prime}-120^{\circ} 56^{\prime} 36^{\prime \prime}$, latitude $37^{\circ} 53^{\prime} 30^{\prime \prime}-38^{\circ} 23^{\prime} 58^{\prime \prime}$ ), the interface between the Yellow Sea and the Bohai Sea.

Many kinds of active substances from echinoderms, such as saponins, polysaccharides, proteins, alkaloids, and fatty acids, have been isolated and reported by scholars both at home and abroad. However, they are mostly extracted from Asteroidea, Holothuroidea, and Echinoidea [2, 3]. These researches on Ophiuroidea from Echinodermata focus more on the morphology, taxonomy, regeneration incidence on species level, wrist-regeneration, and ecological fields $[4,5]$.
Recently, the studies on the preparation and characteristics of the Ophiurasaponin from Ophiura kinbergi have been reported [6]. The four compounds are isolated from Axtroclarus conferus and identified by spectroscopic analysis [7]. However, there has been no report about antioxidant and antimicrobial activities of ophiurasaponin from Ophiopholis mirabilis.

\section{Experiments}

2.1. Extraction and Purification of Ophiurasaponin from Ophiopholis mirabilis. Commonly-used solvent extraction method was applied in the experiment. The marine sample was naturally air-dried and then finely crushed. Afterwards the crushed sample was soaked in $85 \%$ ethanol three times. The collected ethanol-extract was concentrated in rotary evaporator. Equal volume of petroleum ether was added to the concentration extracted six times for degreasing and 
equal volume of $n$-butanol was added to the water extracted six times as well. Then the supernatant was collected and concentrated in the rotary evaporator. After concentration, ophiurasaponin was precipitated by adding 10 times of volume of acetone and then centrifuged. After the precipitate was dried, the crude ophiurasaponin was obtained. Subsequently, some crude ophiurasaponin was purified through AB-8 macroporous resin, eluted with water, 20\% ethanol, $40 \%$ ethanol, $60 \%$ ethanol, and $80 \%$ ethanol in turn. The eluted part of $40 \%$ ethanol was collected, silica gel column chromatography with elution (trichloromethane : methanol: $\mathrm{H}_{2} \mathrm{O}=82: 16: 2$ ), Sephadex LH-20 gel column chromatography with methanol elution, and C18 ODS column chromatography with elution (methanol: $\mathrm{H}_{2} \mathrm{O}=1: 1$ ) were applied to purify ophiurasaponin, and then the refined ophiurasaponin was obtained after being concentrated. The colorimetric method was adopted in this experiment to determinate ophiurasaponin content, compared with the standard saponins.

2.2. Identification of Ophiurasaponin. Liebermann-Burchard reaction, melting test, UV-VIS determination of characteristic absorption peak, and infrared spectrum scanning were used to the identification of ophiurasaponin.

\subsection{Detection of Antioxidation Capabilities}

2.3.1. Determination of the Capacity of Scavenging Hydroxyl Free Radicals. According to the chemiluminescence system of copper sulfate-luminol-vitamin C-hydrogen peroxide [8], Ultra-Weak Luminescence Analyzer was used to detect the capability of the ophiurasaponin scavenging hydroxyl free radicals. The following reagents were added into the sample cell: ophiurasaponin $50 \mu \mathrm{L}(1 \mathrm{mmol} / \mathrm{L})$ (blank sample used as control), ascorbic acid $20 \mu \mathrm{L}(1 \mathrm{mmol} / \mathrm{L})$, luminol $50 \mu \mathrm{L}(1 \mathrm{mmol} / \mathrm{L})$, borate $780 \mu \mathrm{L}(\mathrm{pH} 9.24)$, and hydrogen peroxide $50 \mu \mathrm{L}(1 \mathrm{mmol} / \mathrm{L})$. The analyzers were immediately started and the illumination-intensity was tested within $100 \mathrm{~s}$ under the test conditions of $T=30^{\circ} \mathrm{C}$ and $\mathrm{Hi}-\mathrm{V}:-800 \mathrm{~V}$. The luminescence kinetic curves of hydroxyl inhibited by ophiurasaponin were indicated with illumination time as $X$ axis and illumination intensity integral as $Y$-axis. Origin 7.5 was applied to draw the kinetic curve of inhibited hydroxyl illumination by ophiurasaponin. According to the luminescence kinetic curves, the ratio of the inhibition capacities to ophiurasaponin concentration was calculated, and the value of $\mathrm{IC}_{50}$ was determined as well.

2.3.2. Determination of the Capacity of Scavenging Superoxide Anion Free Radicals. According to the chemiluminescence system of pyrogallol-luminol [9], various concentrations of ophiurasaponin $50 \mu \mathrm{L}$ (blank sample used as control) and pyrogallol $50 \mu \mathrm{L}(6.25 \mathrm{mmol} / \mathrm{L})$ were added into the sample cell. The analyzers were started immediately and the illumination-intensity was tested within $20 \mathrm{~s}$ under the test conditions of $\mathrm{T}=30^{\circ} \mathrm{C}$ and $\mathrm{Hi}-\mathrm{V}$ : $-900 \mathrm{~V}$. The luminescence kinetic curves of superoxide anion inhibited by ophiurasaponin were indicated with illumination time as $X$ axis and illumination intensity integral as $Y$-axis. Origin 7.5 was applied to draw the kinetic curve of inhibited superoxide anion illumination by ophiurasaponin. According to the luminescence kinetic curves, the ratio of the inhibition capacities to ophiurasaponin concentration was calculated, and the value of $\mathrm{IC}_{50}$ was determined as well.

\subsubsection{Determination of the Capacity of Scavenging Hydrogen} Peroxide Free Radicals. According to the chemiluminescence system of hydrogen peroxide-luminol [10], the following reagents were added into the sample cell: various concentrations of ophiurasaponin $50 \mu \mathrm{L}$ (blank sample used as control), luminol $50 \mu \mathrm{L}(1 \mathrm{mmol} / \mathrm{L})$, carbonate buffer $800 \mu \mathrm{L}$ (pH 9.5), and $3 \%$ hydrogen peroxide $100 \mu \mathrm{L}$. The analyzers were started immediately and the illumination-intensity was tested within $100 \mathrm{~s}$ under the test conditions of $T=30^{\circ} \mathrm{C}$ and $\mathrm{Hi}-\mathrm{V}:-850 \mathrm{~V}$. The luminescence kinetic curves of hydrogen peroxide inhibited by ophiurasaponin were indicated with illumination time as $X$-axis and illumination intensity integral as $Y$-axis. Origin 7.5 was applied to draw the kinetic curve of inhibited hydrogen peroxide illumination by ophiurasaponin. According to the luminescence kinetic curves, the ratio of the inhibition capacities to ophiurasaponin concentration was calculated, and the value in $\mathrm{IC}_{50}$ was determined as well.

2.4. Detection of Antimicrobial Activity. The antimicrobial activity of ophiurasaponin was detected by using circular filter paper method [11]. Cell suspensions were finally diluted to $10^{5} \mathrm{CFU} / \mathrm{mL}$ in order to be used in the activity assays. Potato Sucrose Agar (PSA) medium (fungi) and Beef Extract Peptone medium (bacteria) were incubated at $25^{\circ} \mathrm{C}$ for $48 \mathrm{~h}$ (fungi) and $37^{\circ} \mathrm{C}$ for $24 \mathrm{~h}$ (bacteria). Chloramphenicol $(0.04 \mathrm{~g} / \mathrm{mL})$ and miconazole nitrate $(0.04 \mathrm{~g} / \mathrm{mL})$ were used as the positive control and normal saline (NS) was used as the negative control. The minimum inhibitory concentration (MIC) of ophiurasaponin was determined by modified agar dilution method [12]. The concentration of ophiurasaponin was prepared to $0.06 \mathrm{~g} / \mathrm{mL}$, and then it was diluted into $10^{-1}$, $10^{-2}, 10^{-3}, 10^{-4}, 10^{-5}$, and $10^{-6}$, respectively. All the tests were performed in triplicate $(n=3)$ and the results were expressed as $\mathrm{mg} / \mathrm{mL}$.

2.5. Statistical Data Processing. SPSS 11.0 software was used in the single factor analysis of variance by statistically analyzing experimental data, and the results were indicated by the mean standard deviation of plus or minus $(+/-s)$.

\section{Results and Discussion}

3.1. The Chemical Features of Ophiurasaponin. Measured with ultraviolet absorption method, the content of crude ophiurasaponin was $46.75 \%$ and content of the refined ophiurasaponin was $96.72 \%$. Identified by color reaction, ophiurasaponin was determined as steroidal saponins, whose melting points range from $245.4^{\circ} \mathrm{C}$ to $260.6^{\circ} \mathrm{C}$. With ultraviolet visible light scanning within the scope of the $200 \mathrm{~nm}$ to $800 \mathrm{~nm}$ wavelength, compared with the standard saponins, a maximum absorption peak was identified at $276 \mathrm{~nm}$. In 


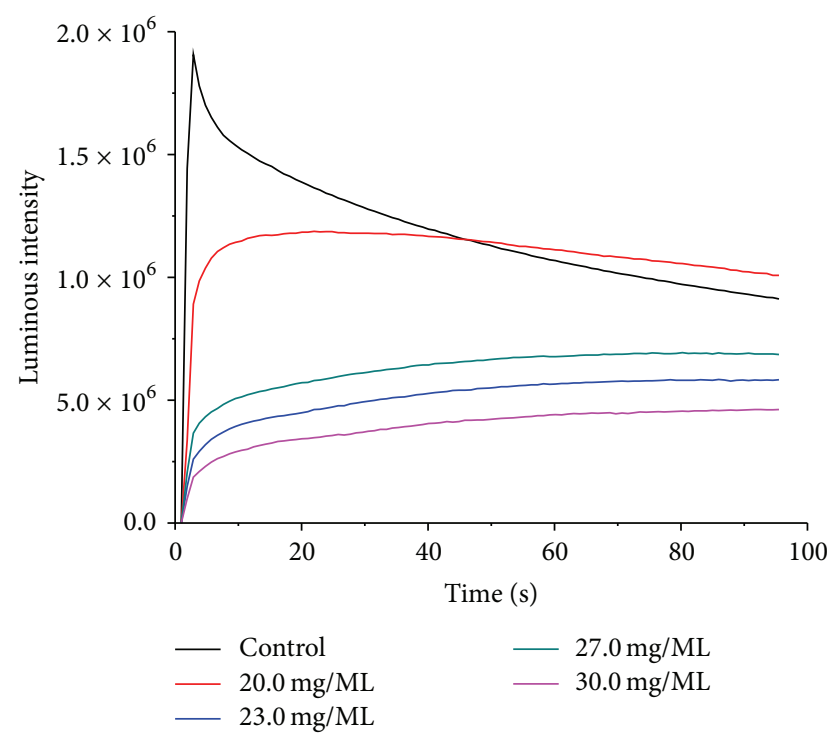

FIGURE 1: Inhibition of different concentrations of ophiurasaponin to hydroxyl: $20 \mathrm{mg} / \mathrm{mL}, 23 \mathrm{mg} / \mathrm{mL}, 25 \mathrm{mg} / \mathrm{mL}, 27 \mathrm{mg} / \mathrm{mL}$, and $30 \mathrm{mg} / \mathrm{mL}$.

$400 \mathrm{~cm}^{-1} \sim 4000 \mathrm{~cm}^{-1}$ range scan, characteristic absorption peak was found similar to the standard saponins in $1030 \mathrm{~cm}^{-1}, 1336 \mathrm{~cm}^{-1}$, and $1610 \mathrm{~cm}^{-1}$. The result suggested that ophiurasaponin and standard saponins were analogous, but ophiurasaponin showed some differences in the fact that Ophiopholis mirabilis came from marine environment and had special structure.

3.2. Antioxidant Capacity. Metabolism of normal physiological process in human body could produce a small quantity of Oxo-free radicalism such as hydroxyl, superoxide anion, and hydrogen peroxide. The amount of free radicals in human body is usually in a homeostasis. A small amount of ROS (reactive oxygen species) free radicals can help transmit energy to maintain the vitality, strengthen the immunity, rid of inflammation, and inhibit tumor, and so forth, while excessive free radicals could interfere with the normal metabolism of human body and lead to sickness [13]. Therefore, the study of free radical scavengers on disease prevention and control is of great significance.

The luminescence kinetic curves of ophiurasaponin inhibiting hydroxyl, superoxide anion, and hydrogen peroxide were shown in Figures 1, 2, and 3, respectively.

The results showed that the antioxidant capabilities increased linearly with the increase of the concentration of ophiurasaponin from Ophiopholis mirabilis in a certain range. The inhibition was constant when it increased to a specific concentration. Usually the concentration of ophiurasaponin at $50 \%$ of illumination inhibition $\left(\mathrm{IC}_{50}\right)$ is taken to measure antioxidant capacity of the samples. Lower $\mathrm{IC}_{50}$ indicates that it has stronger antioxidant capacity.

As shown in Table 1, the result showed that ophiurasaponin from Ophiopholis mirabilis had the strongest inhibition to hydroxyl, the weakest to hydroxyl radicals, and medium to superoxide.

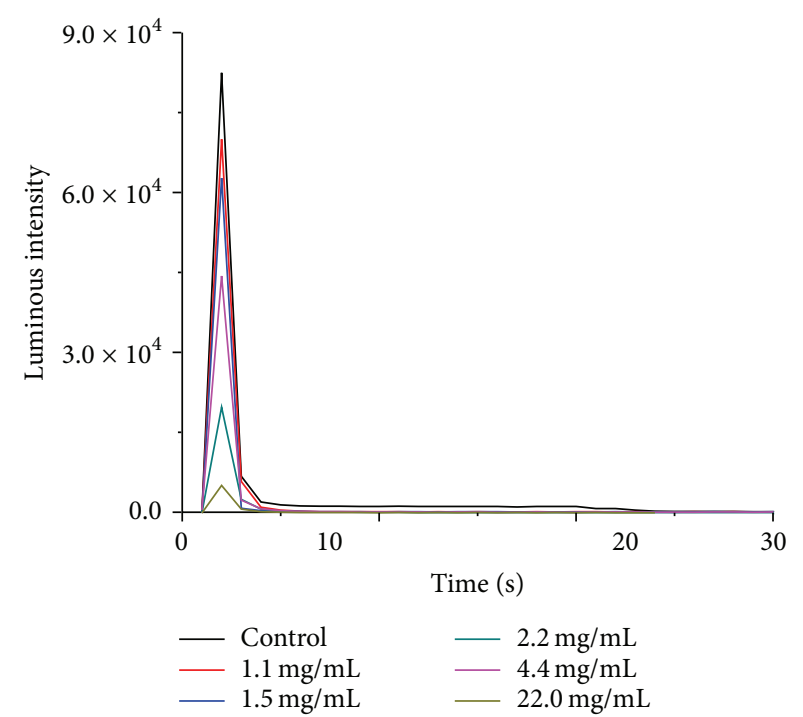

FIGURE 2: Inhibition of different concentrations of ophiurasaponin to superoxide anion: $1.1 \mathrm{mg} / \mathrm{mL}, 1.5 \mathrm{mg} / \mathrm{mL}, 2.2 \mathrm{mg} / \mathrm{mL}, 4.4 \mathrm{mg} / \mathrm{mL}$, and $22 \mathrm{mg} / \mathrm{mL}$.

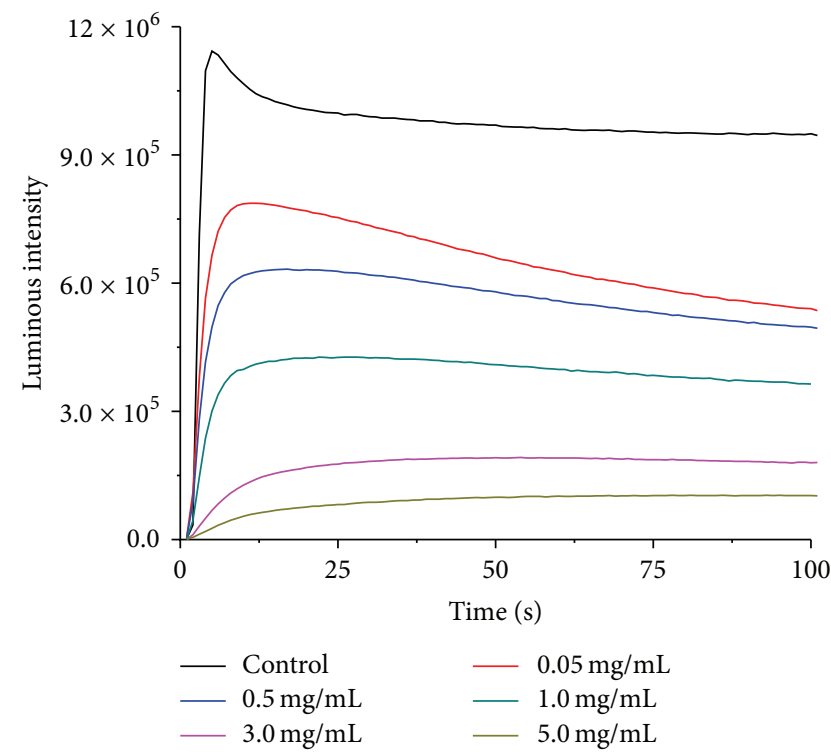

FIGURE 3: Inhibition of different concentrations of ophiurasaponin to hydrogen peroxide: $0.05 \mathrm{mg} / \mathrm{mL}, 0.5 \mathrm{mg} / \mathrm{mL}, 1 \mathrm{mg} / \mathrm{mL}, 3 \mathrm{mg} / \mathrm{mL}$, and $5 \mathrm{mg} / \mathrm{mL}$.

3.3. Results of Antimicrobial Activity. The antimicrobial activity was expressed as the size of the inhibition zone. The larger the size of inhibition zone is, the stronger the antimicrobial activities. As is shown in Table 2, the inhibitory effect on the tested bacteria of refined ophiurasaponin was enhanced significantly compared with the crude. The result showed that the refined ophiurasaponin had stronger antibacterial activity. what is more, the antibacterial ability of Aerobacter aerogenes $(28.2 \mathrm{~mm})$ was close to the positive control $(31 \mathrm{~mm})$, which declared that ophiurasaponin had strong inhibition effect on Aerobacter aerogenes. 
TABLE 1: Capacities of antioxidation of ophiurasaponin $n=6$.

\begin{tabular}{|c|c|c|c|c|c|}
\hline \multicolumn{2}{|c|}{ Superoxide anion } & \multicolumn{2}{|c|}{ Hydroxyl } & \multicolumn{2}{|c|}{ Hydrogen peroxide } \\
\hline $\begin{array}{l}\text { Concentration } \\
(\mathrm{mg} / \mathrm{mL})\end{array}$ & Inhibition (\%) & $\begin{array}{l}\text { Concentration } \\
(\mathrm{mg} / \mathrm{mL})\end{array}$ & Inhibition (\%) & $\begin{array}{l}\text { Concentration } \\
(\mathrm{mg} / \mathrm{mL})\end{array}$ & Inhibition (\%) \\
\hline 22 & $98.32 \pm 0.09$ & 30 & $77.28 \pm 0.10$ & 5 & $94.01 \pm 0.07$ \\
\hline 4.4 & $32.05 \pm 0.15$ & 27 & $59.63 \pm 0.11$ & 3 & $72.14 \pm 0.08$ \\
\hline 2.2 & $19.83 \pm 0.06$ & 25 & $47.45 \pm 0.10$ & 1 & $49.19 \pm 0.11$ \\
\hline 1.5 & $16.32 \pm 0.11$ & 23 & $34.36 \pm 0.05$ & 0.5 & $38.58 \pm 0.06$ \\
\hline 1.1 & $10.17 \pm 0.08$ & 20 & $10.14 \pm 0.13$ & 0.05 & $31.86 \pm 0.12$ \\
\hline The linear & $Y=0.04 x+0.1012$ & \multicolumn{2}{|c|}{$Y=0.0666 x-1.2017$} & \multicolumn{2}{|c|}{$Y=0.1239 x+0.335$} \\
\hline regression & $R^{2}=0.9928$ & \multicolumn{2}{|c|}{$R^{2}=0.9941$} & \multicolumn{2}{|c|}{$R^{2}=0.9918$} \\
\hline $\mathrm{IC}_{50}(\mathrm{mg} / \mathrm{mL})$ & $9.98 \pm 0.12$ & \multicolumn{2}{|c|}{$25.54 \pm 0.07$} & \multicolumn{2}{|c|}{$1.37 \pm 0.03$} \\
\hline
\end{tabular}

TABLE 2: Inhibition of ophiurasaponin from Ophiopholis mirabilis to bacteria $n=3$.

\begin{tabular}{lccccc}
\hline Species & $\begin{array}{c}\text { Positive control } \\
\text { (chloramphenicol) }\end{array}$ & $\begin{array}{c}\text { Crude } \\
(\mathrm{mm})\end{array}$ & $\begin{array}{c}\text { Ophiurasaponin } \\
\text { MIC }(\mathrm{mg} / \mathrm{mL})\end{array}$ & $\begin{array}{c}\text { Refined } \\
(\mathrm{mm})\end{array}$ & $\begin{array}{c}\text { Ophiurasaponin } \\
\text { MIC }(\mathrm{mg} / \mathrm{mL})\end{array}$ \\
\hline Bacillus subtilis & $44.5 \pm 0.02$ & $12.2 \pm 0.10$ & $1.1 \pm 0.05$ & $26.9 \pm 0.01$ & $0.0443 \pm 0.03$ \\
Escherichia coli & $51.0 \pm 0.04$ & $11.5 \pm 0.08$ & $1.1 \pm 0.03$ & $32.5 \pm 0.03$ & $0.0443 \pm 0.03$ \\
Staphylococcus aureus & $46.0 \pm 0.06$ & $11.2 \pm 0.07$ & $0.11 \pm 0.01$ & $30.9 \pm 0.09$ & $0.0443 \pm 0.07$ \\
Proteusbacillus vulgaris & $48.0 \pm 0.05$ & $11.2 \pm 0.01$ & - & $29.6 \pm 0.05$ & - \\
Aerobacter aerogenes & $31.0 \pm 0.06$ & $16.0 \pm 0.05$ & $1.1 \pm 0.01$ & $28.2 \pm 0.04$ & $0.0443 \pm 0.01$ \\
\hline
\end{tabular}

Note: - : no inhibitory effect.

TABLE 3: Inhibition of ophiurasaponin from Ophiopholis mirabilis on fungi $n=3$.

\begin{tabular}{|c|c|c|c|c|c|}
\hline Species & $\begin{array}{c}\text { Positive control } \\
\text { (miconazole nitrate) }\end{array}$ & $\begin{array}{l}\text { Crude } \\
(\mathrm{mm})\end{array}$ & $\begin{array}{l}\text { Ophiurasaponin } \\
\text { MIC }(\mathrm{mg} / \mathrm{mL})\end{array}$ & $\begin{array}{l}\text { Refined } \\
(\mathrm{mm})\end{array}$ & $\begin{array}{c}\text { Ophiurasaponin } \\
\text { MIC }(\mathrm{mg} / \mathrm{mL})\end{array}$ \\
\hline Penicillium digitatum & $21.4 \pm 0.03$ & - & - & - & - \\
\hline Mucor circinelloides & $30.7 \pm 0.02$ & $11.5 \pm 0.06$ & $1.1 \pm 0.03$ & $13.0 \pm 0.02$ & $4.43 \pm 0.02$ \\
\hline Aspergillus flavus & $28.7 \pm 0.01$ & - & - & - & - \\
\hline Saccharomyces cerevisiae & $20.7 \pm 0.04$ & - & - & - & - \\
\hline Saccharomyces cerevisiae Hansen & $19.8 \pm 0.03$ & $11.2 \pm 0.04$ & $1.1 \pm 0.04$ & - & - \\
\hline
\end{tabular}

Note: - : no inhibitory effect.

As shown in Table 3, ophiurasaponin from Ophiopholis mirabilis could inhibit Mucor circinelloides proliferation; however, it had no inhibition on Penicillium digitatum, Aspergillus flavus, and Saccharomyces cerevisiae. The result showed thatcrude ophiurasaponin inhibited the growth of Saccharomyces cerevisiae Hansen, but the refined ophiurasaponin did not inhibit its growth, because saponins of other substances in crude ophiurasaponin may be reactive. In addition, theinhibition of ophiurasaponin from Ophiopholis mirabilis on fungi was much less effective than that on bacteria.

The minimum inhibitory concentration (MIC) was defined as the lowest concentration of ophiurasaponin that inhibited growth. The MIC value of ophiurasaponin was evidently demonstrated in Table 3. The MIC of refined ophiurasaponin was $0.0443 \mathrm{mg} / \mathrm{mL}$, much lower than that of the crude on bacterial. Staphylococcus aureus was the most sensitive bacteria to ophiurasaponin, the MIC value of the crude ophiurasaponin was $0.11 \mathrm{mg} / \mathrm{mL}$, and the MIC value of the refined ophiurasaponin was $0.0443 \mathrm{mg} / \mathrm{mL}$. Mucor circinelloides was less sensitive to ophiurasaponin ( $4.43 \mathrm{mg} / \mathrm{mL} \mathrm{MIC})$. Ophiurasaponin was not sensitive to Proteusbacillus vulgaris, Penicillium digitatum, Aspergillus flavus, and Saccharomyces cerevisiae. The result illuminated that the antimicrobial ability of ophiurasaponin had a certain selectivity and the antimicrobial ability of ophiurasaponin had a stronger effect on bacteria.

\section{Conclusions}

The paper showed that ophiurasaponin extracted from Ophiopholis mirabilis had excellent antioxidant activity and inhibition on microbial activity. Although further studies are needed to better evaluate its activities and mechanism(s), our 
data suggest that ophiurasaponin might be a candidate for the antioxidant and antimicrobial marine drugs.

\section{Conflict of Interests}

The authors declare that there is no conflict of interests regarding the publication of this paper.

\section{Acknowledgments}

This work was supported by the Shandong province Natural Science Foundation (no. ZR2013DM002). The authors thank Professor Qiusheng Zheng and Huiling Leng for their critical reading of this paper.

\section{References}

[1] Y. L. Liao, Fauna Sinica Invertebrata Echinodermata Ophiuroidea, vol. 40, Science Press, Beijing, China, 2004.

[2] F. Kisa, K. Yamada, T. Miyamoto, M. Inagaki, and R. Higuchi, "Constituents of holothuroidea, 17. Isolation and structure of biologically active monosialo-gangliosides from the sea cucumber Cucumaria echinata," Chemical and Pharmaceutical Bulletin, vol. 54, no. 7, pp. 982-987, 2006.

[3] K.-S. Nam and Y.-H. Shon, "Chemopreventive effects of polysaccharides extract from Asterina pectinifera on HT-29 human colon adenocarcinoma cells," BMB Reports, vol. 42, no. 5, pp. 277-280, 2009.

[4] R. Bannister, I. M. McGonnell, A. Graham, M. C. Thorndyke, and P. W. Beesley, "Afuni, a novel transforming growth factor- $\beta$ gene is involved in arm regeneration by the brittle star Amphiura filiformis," Development Genes and Evolution, vol. 215, no. 8, pp. 393-401, 2005.

[5] T. Fujita and S. Ohta, "Spatial structure within a dense bed of the brittle star Ophiura sarsi (Ophiuroidea: Echinodermata) in the bathyal zone off otsuchi, Northeastern Japan," Journal of the Oceanographical Society of Japan, vol. 45, no. 5, pp. 289-300, 1989.

[6] C. H. Guo, H. Z. Jin, X. J. Ni et al., "Preparation and primary identification of ophiurasaponin from Ophiura kinbergi," Current Zoology, vol. 47, pp. 131-133, 2001.

[7] C. W. Lin, J. Y. Su, L. M. Zeng et al., "Chemical constituents of axtroclarus conferus," Journal of Instrumental Analysis, vol. 7, pp. 59-61, 2002.

[8] S. Hanaoka, J.-M. Lin, and M. Yamada, "Chemiluminescence behavior of the decomposition of hydrogen peroxide catalyzed by copper(II)-amino acid complexes and its application to the determination of tryptophan and phenylalanine," Analytica Chimica Acta, vol. 409, no. 1-2, pp. 65-73, 2000.

[9] L. Jian, "Increased carbon disulfide-stimulated chemiluminescence in the pyrogallol-luminol system," Luminescence, vol. 16, no. 4, pp. 281-283, 2001.

[10] F. J. Pérez and S. Rubio, "An improved chemiluminescence method for hydrogen peroxide determination in plant tissues," Plant Growth Regulation, vol. 48, no. 1, pp. 89-95, 2006.

[11] H. D. Chludil, A. M. Seldes, and M. S. Maier, "Antifungal steroidal glycosides from the Patagonian starfish Anasterias minuta: structure-activity correlations," Journal of Natural Products, vol. 65, no. 2, pp. 153-157, 2002.
[12] R. Kumar, A. K. Chaturvedi, P. K. Shukla, and V. Lakshmi, "Antifungal activity in triterpene glycosides from the sea cucumber Actinopyga lecanora," Bioorganic and Medicinal Chemistry Letters, vol. 17, no. 15, pp. 4387-4391, 2007.

[13] H. Ahsan, A. Ali, and R. Ali, "Oxygen free radicals and systemic autoimmunity," Clinical and Experimental Immunology, vol. 131, no. 3, pp. 398-404, 2003. 

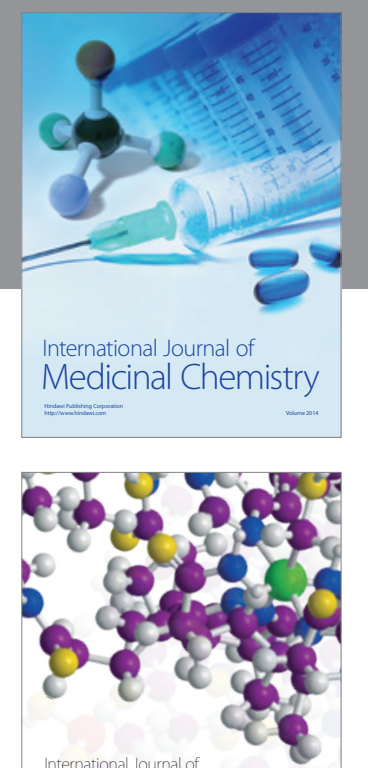

\section{Carbohydrate} Chemistry

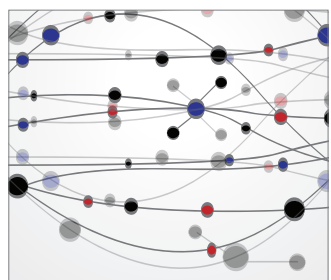

The Scientific World Journal
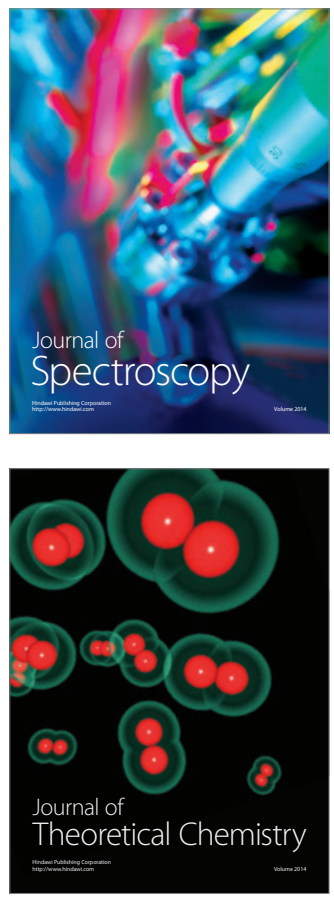
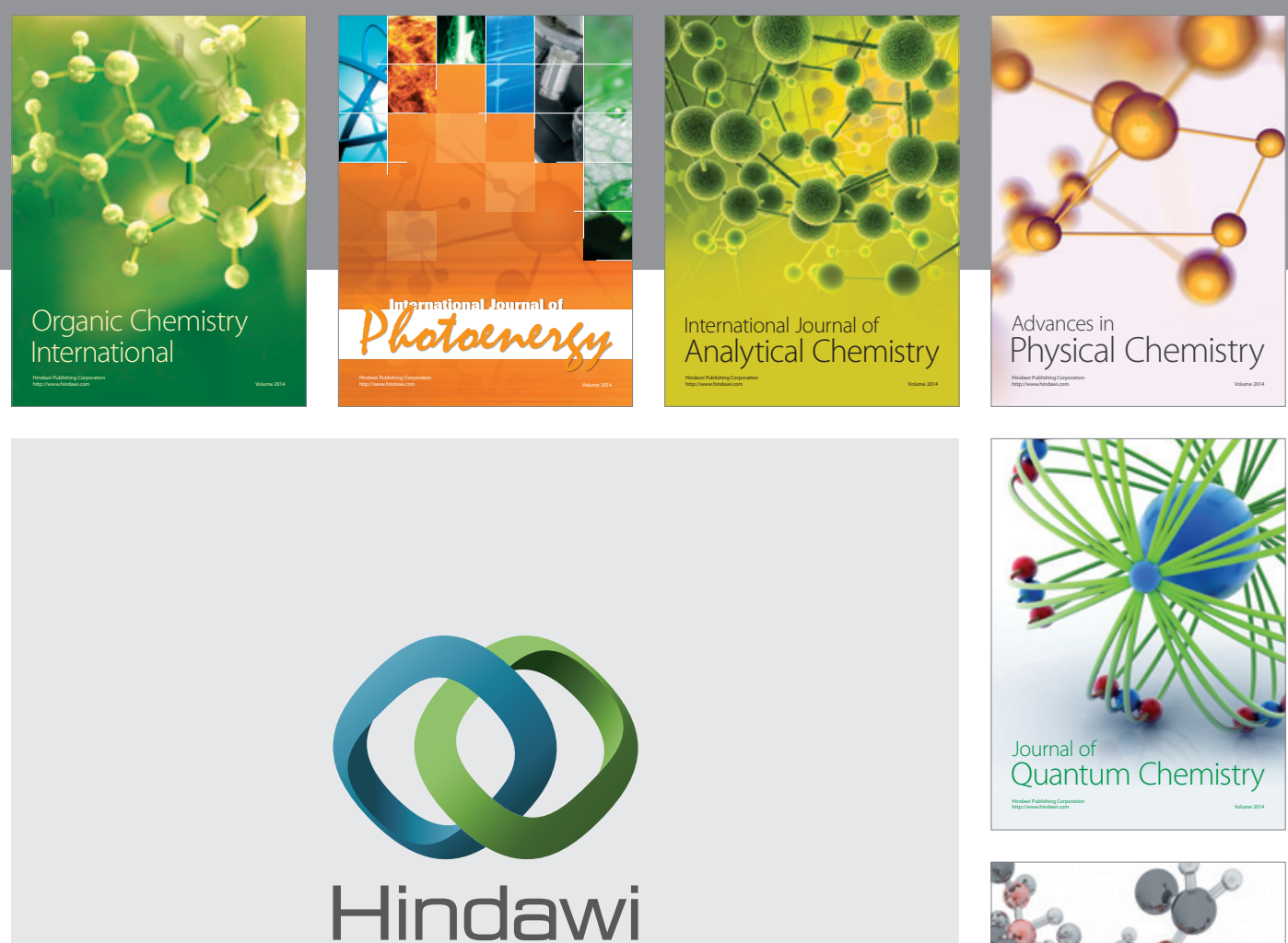

Submit your manuscripts at

http://www.hindawi.com

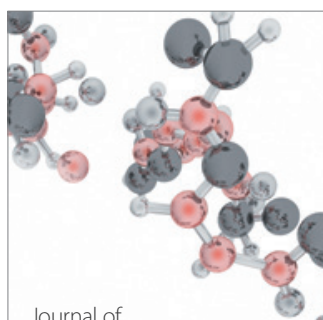

Analytical Methods

in Chemistry

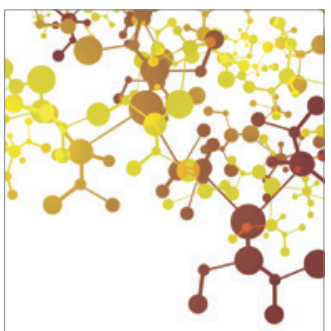

Journal of

Applied Chemistry

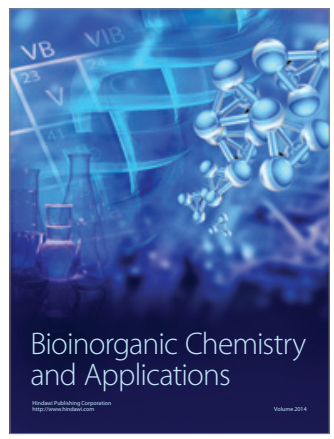

Inorganic Chemistry
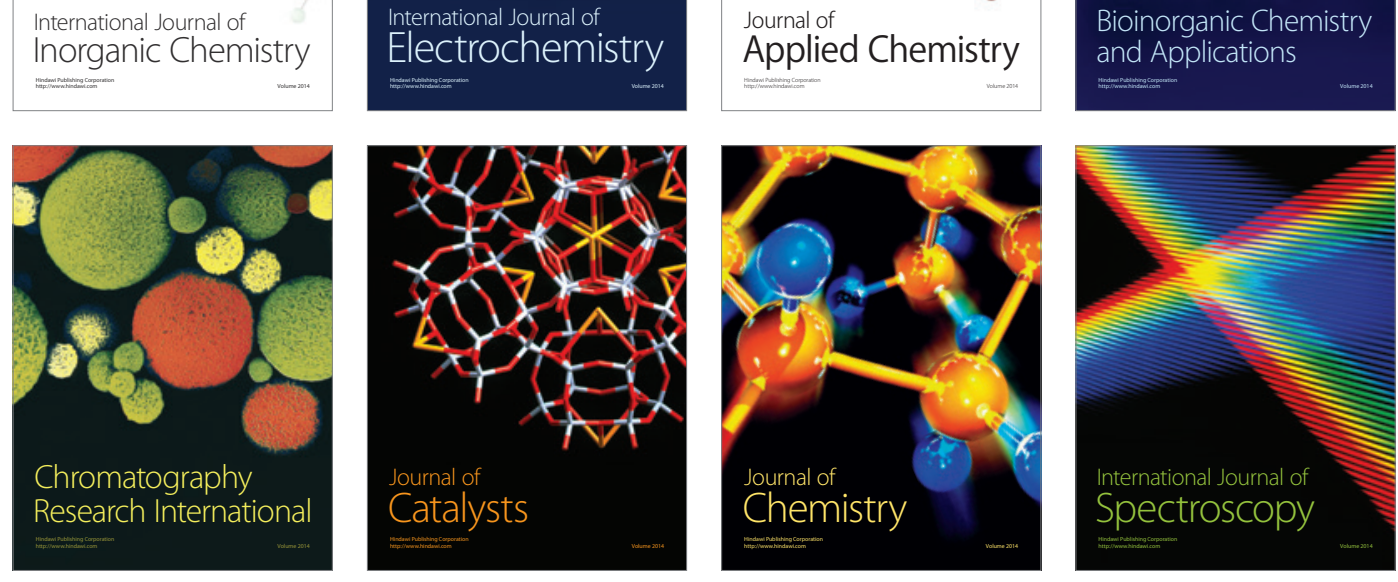